\title{
Precision Agriculture: A Remote Sensing Monitoring System Architecture ${ }^{\dagger}$
}

\author{
Anna Triantafyllou *(D), Panagiotis Sarigiannidis* $*$ and Stamatia Bibi * \\ Department of Electrical and Computer Engineering, University of Western Macedonia, Kozani 50100, Greece \\ * Correspondence: atriantafyllou@uowm.gr (A.T.); psarigiannidis@uowm.gr (P.S.); sbibi@uowm.gr (S.B.) \\ + This paper is an extended version of our paper published in International Workshop on IoT Applications and \\ Industry 4.0 (IoT4 2019), co-located with IEEE DCOSS 2019, Santorini Island, Greece.
}

Received: 29 September 2019; Accepted: 3 November 2019; Published: 9 November 2019

\begin{abstract}
Smart Farming is a development that emphasizes on the use of modern technologies in the cyber-physical field management cycle. Technologies such as the Internet of Things (IoT) and Cloud Computing have accelerated the digital transformation of the conventional agricultural practices promising increased production rate and product quality. The adoption of smart farming though is hampered because of the lack of models providing guidance to practitioners regarding the necessary components that constitute IoT-based monitoring systems. To guide the process of designing and implementing Smart farming monitoring systems, in this paper we propose a generic reference architecture model, taking also into consideration a very important non-functional requirement, the energy consumption restriction. Moreover, we present and discuss the technologies that incorporate the seven layers of the architecture model that are the Sensor Layer, the Link Layer, the Encapsulation Layer, the Middleware Layer, the Configuration Layer, the Management Layer and the Application Layer. Furthermore, the proposed Reference Architecture model is exemplified in a real-world application for surveying Saffron agriculture in Kozani, Greece.
\end{abstract}

Keywords: Wireless Sensor Networks; Internet of Things; precision agriculture; smart farming; communication technologies; Cloud Computing

\section{Introduction}

Presently, the digital transformation of the agricultural sector is considered a priority to face the numerous challenges presented in the fields. Environmental monitoring and remote controlling in agriculture is rapidly growing towards developing more productive and competitive agricultural systems and tools. Precision Agriculture and Smart Farming can lead to this direction. These two terms refer to the integration of advanced technologies into existing agricultural practices to achieve fine-grid crops management. Smart farming systems can provide to farmers meaningful environmental data from the cultivation fields aiming to boost competitiveness and profit. Almost every aspect of the agricultural field can benefit from these kinds of technological advances ranging from planting and irrigation processes to plant protection and harvesting methods.

The future of precision agriculture lies upon modern technological advancements and remote sensing techniques using Unmanned Aerial Vehicles (UAV) and different kind of smart sensors. Sensors are measuring devices that convert an external stimulus or else input signal, into an appropriately measurable output signal. Sensor devices can transform a macroscopic size (light, power, pressure, etc.) to an electrically measurable size. Once the electrical signal is processed, it is converted into a standardized signal with certain characteristics. The sensor's properties can be altered in a measurable manner, either directly or indirectly by the exposure to a particular analyzer or change in environmental conditions. UAVs are flying vehicles that do not have a pilot on their spindle. Instead they fly either 
autonomously or by means of remote control. Unmanned aircraft used for remote monitoring are part of Unmanned Aerial Systems (UASs). UASs involve all necessary devices and procedures for UAV operation, while managing data collection. UAVs enable Earth Observation (EO) towards improving accuracy, executing more frequent and better monitoring of the fields and cover large (not easily accessible) areas. Sensors and UAVs are a part of the Internet of Things (IoT) paradigm. The IoT is a modern communication network involving the employment of a vast number of distributed smart devices around a unified wide area network. Its basic characteristic stands upon the ability to recognize and notify users instantly about real-time events by the use of smart objects. Smart objects-devices have basic computational skills, constrained resources and unique identifiers for communication.

The adoption of smart farming though is hampered because of the lack of models providing guidance to practitioners regarding the necessary components that constitute IoT-based monitoring systems. The typically dense and heterogeneous nature of IoT deployments poses immense challenges on interoperability among the desired components. However, an efficiently designed architecture based on modern enhanced IoT technologies can pave the way into easily adopting and deploying smart systems into our everyday life activities. The latest years a significant number of efforts were presented regarding the most suitable network structure and technologies for smart farming applications [1-9]. However, the architectural models presented in [1,7-9] were based only on static sensor nodes, ignoring the advantage of UAVs in monitoring and failing to provide efficient mapping of the field to the farmer. Furthermore, no prediction mechanisms were employed in [2] towards improving production. Moreover, limited contribution was provided in [3-5] regarding energy saving and link layer IoT technologies in farm management information systems, while the data-driven agricultural model proposed in [6] did not discuss data security issues. In contrast to previous studies, the contribution of this paper lies upon the presentation of a detailed architectural model for an advanced smart farming monitoring system by using UAVs and taking into consideration energy-saving and security requirements. This architectural model engages novel IoT technologies [10] and Wireless Sensor Networks (WSNs) capabilities to provide a sufficient view of precision agriculture. Furthermore, the proposed architecture enables a combination of modern remote sensing techniques such as UAV tracking, Global Positioning System (GPS) for location detection, Geographic Information Systems (GIS), real-time monitoring with different types of sensors and intelligent input control systems. These technologies have already been tested in various agricultural fields in different countries for the cultivation of rice, wheat, tomatoes, vegetables, potatoes, ornamental flowers, chilly, cacao, pepper, corn, olives, apples, lemons, grape and others. By incorporating new technologies into agricultural production and by using modern EO techniques growers will be able to manage their crops at a different and more advanced kind of level in detail that was not possible a few years ago. This paper is an extension of our work in [11]. Towards enhancing contribution a use case study is also presented regarding an ongoing research project known as Drone Innovation in saffron Agriculture Surveillance(DIAS). The DIAS architectural model is based on the proposed paradigm.

This paper is organized as follows. Section 2 presents an overview of the proposed smart farming monitoring system architecture. In Section 3, the sensor layer is introduced, followed by the network layer and its suitable protocols and technologies regarding communication, routing, encapsulation techniques and interoperability mechanisms in Section 4. Section 5 is focused on the management layer and the provided services of the proposed monitoring system, while Section 6 focuses on IoT agricultural applications. Section 7 deals with energy-saving technologies and security mechanisms that can be implemented in cooperation with networking technologies of the system. The use case study of the DIAS architecture is presented in Section 8. Existing challenges are mentioned and discussed in Section 9. Finally, Section 10 concludes this study.

\section{The Architecture of a Smart Farming Monitoring System}

A precision agriculture monitoring system consists mainly of the sensing agricultural parameters, the identification of sensing location and data gathering, the routing of data from crop field to 
control station for decision making, the actuation and control decision based on sensed data and the visualization of results to the grower through an application. The architectural design of our model follows the ISO/IEC 7498-1 standard or else known as the OSI Model [12], proposing a communication system into seven abstraction layers. However, due the employment of IoT technologies and artificial intelligence capabilities the basic agricultural layers are defined as presented in Figure 1:

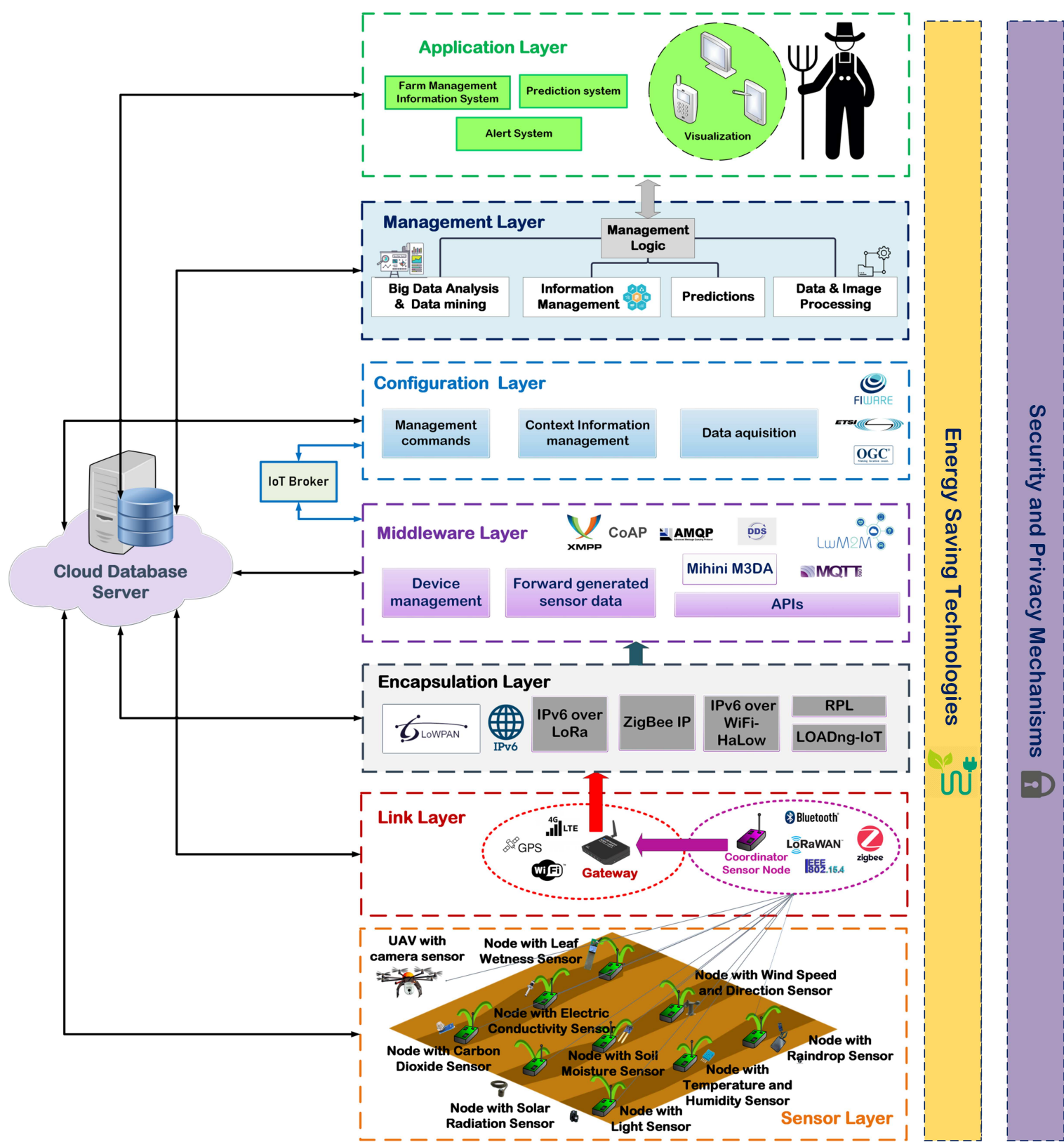

Figure 1. Remote Sensing System Architecture.

- The Sensor Layer, referring to the Physical Layer of the OSI Model, includes all kinds of crops sensors and smart objects for data collection and monitoring. Sensors can be placed under ground(in the soil), on the crops or on UAVs [6]. Underground sensors are especially manufactured to be water resistant and usually refer to measurements of moisture, $\mathrm{pH}$ and soil chemical properties such as sulfur. UAV sensors measure environmental parameters such as humidity, temperature, wind speed, luminosity or solar radiation. However, the most popular kind of sensors to be placed on UAVs are thermal cameras. Thermal drones which use vision 
imaging cameras have so many positive uses by detecting heat coming from almost all objects and materials turning them into images and video.

- The Link Layer, referring to the Data Link Layer of the OSI Model, constitutes of all available networking and routing technologies between sensors for information exchange. To deploy efficient crop and field management the IoT platform uses Wireless Sensor Networks (WSNs). The use of WSN in smart farming systems provides immediate monitoring and optimization of crop quality, while offering a potential for large area surveillance with high sampling densities. The constant monitoring of a great number of environmental parameters by distributed sensor nodes along the field help the grower supervise and maintain optimal conditions to achieve maximum productivity with remarkable energy savings.

- The Encapsulation Layer, referring to the Network Layer of the OSI Model, focuses on the establishment of smart sensor connection to the IPv6-based internet. This layer consists of IoT networking encapsulation techniques and routing protocols to transform the regular WSN network traffic into smart information. In other words, the technologies of this layer enable the cultivated field sensory data to be encapsulated in IPv6 routing packets and be forwarded to the according network server.

- The Middleware Layer, referring to the Transport Layer of the OSI Model, uses different application level transport protocols in order to forward the data generated from IoT sensor devices based on different paradigms. It also provides interfaces that enable device communication for management or actuation purposes. This layer facilitates the desired interoperability due to the existence of diverse standards, which are endorsed by different entities.

- The Configuration Layer, referring to the Session and Presentation Layers of the OSI Model, is situated between the Middleware Layer and the Management Layer. This layer is responsible for gathering the raw data coming from the devices or other external services, curate, harmonize and possibly aggregate them, so that they can be published as context information, or supplied to upstream data processing algorithms or analytics. In addition, this layer is also capable of sending actuation commands to the Middleware Layer. Finally, the Configuration Layer may also be capable of gathering data from other data sources, such as agricultural machinery or public geo-services.

- The Management Layer involves the processing and analysis of the collected data. In this layer the most efficient data management and data mining techniques are adopted to obtain accurate predictions and support regarding field operations such as optimized pesticide application, disease detection, efficient irrigation management. Data processing is supported by Decision Support Systems (DSS) that take care of the overall management of available collected information from the fields towards increasing productivity, optimizing crop yield, maintaining quality and saving resources. It is well known that farmers suffer great economic losses due to incorrect weather forecasting or incorrect irrigation methods. Data analysis is the most important component of IoT agricultural systems resulting in efficient pesticide use and protection against diseases. This layer can be considered to be an additional layer regarding the OSI Model enabling artificial intelligence advancements to the overall system.

- The Application Layer, referring to the Application Layer of the OSI Model, includes all suitable application module interfaces for implementing fertilizer and irrigation control, disease and animal detection, alerts regarding the cultivation process and visualization of statistical data. This layer enables the farmer to monitor and manage his fields in a user-friendly way. Data visualization techniques such as graphs, heatmaps, orthomosaics, and three-dimensional models are employed, among others, to allow easy and intuitive representation of the knowledge acquired from the field monitoring. The farmer can inspect the results produced by the services of the system and take action accordingly. 
Energy-saving mechanisms and data privacy and security techniques are also considered to be very important in the context of Smart farming and should be applied vertically in all the layers of the architecture. To support the efficiency and effectiveness of a smart farming monitoring system energy consumption should be kept under control. Due to the limited battery life and constrained resources of sensor nodes, energy-saving techniques must be applied across the sensor and network layer accordingly. Energy-saving techniques deal with the active and inactive operational time in each sensor node, the scheduling of information transmission and the routing process of data packets. Moreover, security mechanisms are vital for maintaining the privacy of the collected data and safeguarding farmers' personal information exchange.

\section{The Sensor Layer}

The Sensor Layer is in charge of acquiring the data of the different climatic and soil variables involved in the growth and production of the crops. Data can be acquired either by ground sensors, located above or inside the soil, or by UAV sensors involving embedded cameras, location and other sensors. The Sensor Layer constitutes the cradle of modern EO approaches towards optimizing decision support in remote sensing monitoring systems. EO monitoring of cultivation areas is highly enhanced using autonomous UAVs. Popular EO techniques include satellite and radar-based technologies [13] towards estimating basic biophysical parameters in the fields, such as Leaf Area Index (LAI), crop height and water requirements [14,15]. According to [16] radar-based technologies are used to enable the estimation of soil moisture spatial variability and can efficiently estimate LAI. However, EO can achieve sturdy higher spatial resolutions only with calibration against accurate ground truth instruments that measure at within field scale resolutions. UAVs deploy EO via using photogrammetry techniques enabling the generation of three-dimensional digital surface models of the fields. In particular, autonomous, manual or GPS-based image acquisition, ortho-image generation and image triangulation and geo-referencing based on navigation sensors are employed. More information regarding these methods is provided in Sections 5 and 8.2. In the Sensor Layer each sensor sends the acquired data in the cloud through a WSN. The WSN is made up of sensor nodes that operate under a mesh or point-to-point topology, a coordinator node and a gateway. Each node in such a network is connected to one or more sensors [17]. In precision agriculture the most important types of sensors for measuring the different types of corps attributes are:

- Optical Sensors/UAV Sensors: Optical sensors are usually embedded in UAVs and use light reflection information to measure the varying properties of soil and vegetation. In that case, the sensors acquire image data, which are further analyzed with photogrammetry techniques. Object detectors and pattern recognition form the basic building block for extracting information from the images. Such information may involve the vegetation and soil color, the moisture content and temperature of soil and vegetation, the position, height, size and shape of vegetation along with the level of chlorophyll. In this category we find visible light sensors, multispectral sensors, hyperspectral sensors and thermal sensors.

- Electrochemical Sensors/Ground Sensors: These types of sensors acquire data regarding the nutrient contents of soil and its associated $\mathrm{pH}$. Electrodes in these sensors work by detecting specific ions in the soil. Different families of electrochemical sensors can be recognized depending on the electrical magnitude used for transduction of the recognition event: potentiometric, which indicates change of membrane potential; conductometric, which indicates change of conductance; impedimetric, which indicates change of impedance; and voltammetric or amperometric, which indicates change of current for an electrochemical reaction with the applied voltage in the first case, or with time at a fixed applied potential in the latter.

- Location Sensors/UAV Sensors: Location sensors are usually embedded in UAVs and provide spatial information regarding the positioning of an element. These types of sensors use signals from GPS satellites to determine latitude, longitude, and altitude to within feet. Three satellites minimum are required to triangulate a position. Precise positioning is the cornerstone of precision 
agriculture. GPS integrated circuits such as the NJR NJG1157PCD-TE1 are a good example of location sensors.

- Weather Stations/Ground Sensors: Weather stations are free-standing units situated at different locations throughout the cultivating fields. These stations measure various data for precision agriculture such as airflow, seasonal rainfall, speed of wind, humidity level, direction of wind, atmospheric pressure and solar radiation, etc. Weather stations are an important component of EO technologies since they can provide daily agro-meteorological information regarding the cultivating fields.

Summarizing the above, Table 1 presents the most frequently acquired data by sensors in the agricultural domain. A sensor node consists of a radio transceiver with an internal antenna or a connection to an external antenna, a micro-controller, an electronic circuit for interfacing with the sensors and an energy source, usually a battery or a built-in energy harvested form. There are numerous commercial models of micro-controllers to be used in precision agriculture applications. The most popular ones are the Arduino, the Raspberry Pi, the Atmega328 and the LPC2148 boards. Accordingly, commonly used wireless communication modules used are the XBee module, the WSN802G module and the NRF24L01 module. A sensor node can vary in size and cost, depending on the complexity of its capabilities. Size and cost constraints result in corresponding limitations on resources such as energy, memory, computing speed, and bandwidth of communications. The types of sensors that are mostly used in Smart farming monitoring systems are summarized in Table 2.

Table 1. Precision agriculture sensor data types.

\begin{tabular}{cc}
\hline Data Type & Sensor Type \\
\hline Soil moisture and temperature & Ground sensors \\
\hline Soil color & UAV sensors \\
\hline Environmental humidity and temperature & Ground sensors or UAV sensors \\
\hline Leaf-wetness & Ground sensors or UAV sensors \\
\hline Electric conductivity & Electrochemical sensors \\
\hline Wind speed and direction & Weather stations \\
\hline Barometric pressure & Weather stations \\
\hline Carbon dioxide & Electrochemical sensors \\
\hline Ph value & Electrochemical sensors \\
\hline Light intensity & Weather stations or Ground sensors \\
\hline Solar radiation & Weather stations or Ground sensors \\
\hline Rainfall & Weather stations \\
\hline Size of crops & UAV sensors \\
\hline Shape of crops & UAV sensors \\
\hline Thickness of plant stem & UAV sensors \\
\hline Latitude, longitude and altitude of the plants & Location sensors \\
\hline
\end{tabular}


Table 2. Precision agriculture sensor models.

\begin{tabular}{cc}
\hline Sensor Type & Sensor Model \\
\hline Soil moisture sensor & 10-HS,SY-HS-220, FC-28 \\
\hline Temperature sensor & LM35, SHT15, DS18B20 \\
\hline Humidity sensor & DHT22, DHT11 \\
\hline Electric conductivity sensor & DFR0300 \\
\hline Wind speed and direction sensor & SEN0170 \\
\hline Barometric pressure sensor & BMP180 \\
\hline Carbon dioxide sensor & CDM4161A, MHZ16 \\
\hline Ph sensor & MCP1525 \\
\hline Light sensor & TSL2561, BH1750 \\
\hline Solar radiation sensor & 6450 TSR \\
\hline Thermal sensors & ThermoMAP
\end{tabular}

\section{The Network Layer}

\subsection{The Link Layer}

In precision agriculture WSN communication protocols and technologies are used to support the connection between sensor nodes in the network and to provide a channel for communication between the coordinator node and the gateway. According to the type of application, such as precision farming, field irrigation management or greenhouse crop management, the sensor network topology and communication demands may differ. Hardware and software characteristics may also affect the choice of communication technology to be used between the nodes. Each node uses a routing protocol [18] in the view of transferring the data collected to the coordinator node.

Based on many experimental studies on agricultural fields, there is not an ideal combination of a specific communication technology and a routing protocol. Discovering and keeping up with routes in WSNs is a quite demanding task since energy restrictions and alterations in node status, such as failure may cause sudden changes in the network topology. It is a fact that the wireless routing solution for agriculture applications should be highly energy-efficient, scalable, and autonomous. Up until now routing tactics proposed in the literature for WSNs employ specific methods such as data aggregation, clustering, different node role assignment and data-centric methods.

The basic goal is to build each smart monitoring system upon application appropriate networking technologies to operate efficiently with minimum energy consumption. Once the coordinator node obtains the data it forwards the flow of information to the gateway to reach the main server, where the database is located. However, in some cases the coordinator node can be substituted by a base station to obtain the collected data using a Wi-Fi connection as presented in [2], or another cellular communication technology.

\subsubsection{Precision Agriculture Communication Protocols}

There is a wide variety of networking technologies suitable for the deployment of smart farming applications. The most popular are the following:

- The IEEE 802.15.4 standard is a widely used networking technology in precision agriculture and defines the physical layer and the Media Access Control (MAC) technique in Low-Rate Wireless Personal Area Networks (LR-WPANs).

- ZigBee is another suitable technology for short range radio communication in the fields using low-power devices capable of transmitting data over long distances using intermediate stations. 
- LoRa is a type of wireless configuration that has been created to achieve long-range connections for Low-power Wide Area Networks (LPWANs). LoRAWAN is a protocol for managing communication between LPWAN gateways and nodes.

- Bluetooth Low Energy is a global personal area network protocol built for transmitting small data pieces infrequently at low rates with significantly low power consumption per bit.

- RFID (Radio Frequency Identification) is a different technology that uses radio signals to monitor and identify in real time objects without requiring line-of-sight communication. An RFID system includes a reader, a tag, and a host and is presented as ideal for field monitoring in multiple studies.

Moreover, the communication between sensor nodes and a base station can be supported by:

- $\quad$ the Wi-Fi protocol, based on the IEEE 802.11 standard. This standard specifies the set of media access control (MAC) and physical layer (PHY) protocols for implementing wireless local area network (WLAN) Wi-Fi computer communication in various frequencies.

- the GSM (Global System for Mobile Communications), a standard developed by the European Telecommunications Standards Institute (ETSI) to describe the protocols for second-generation $(2 \mathrm{G})$ digital cellular networks used by mobile devices such as mobile phones and tablets.

- the GPRS (General Packet Radio Service) technology standard that provides rapid sending and receiving of data over the GSM mobile networks based on packet switching, a well-known network transmission process.

- the $2 \mathrm{G}, 3 \mathrm{G}$ and $4 \mathrm{G}$ (LTE) are respectively the 2nd, 3rd and 4th generation of GSM technology aiming at higher speeds.

Table 3 summarizes the communication technologies adopted in smart farming systems according to literature.

Table 3. Smart farming networking technologies.

\begin{tabular}{ccccc}
\hline Communication Technology & Data Rate & Frequency Band & Range & References \\
\hline IEEE 802.15.4 & $20-250 \mathrm{Kbps}$ & $2400 / 915 / 868 \mathrm{MHz}$ & $10 \mathrm{~m}$ & {$[7]$} \\
IEEE 802.15.4-ZigBee & $20-250 \mathrm{Kbps}$ & $2400 / 915 / 868 \mathrm{MHz}$ & $10-100 \mathrm{~m}$ & {$[19]$} \\
Wi-Fi-IEEE 802.11 & $450 \mathrm{Mbps}$ & $2.4 \mathrm{GHz}-5 \mathrm{GHz}$ & $100 \mathrm{~m}$ & {$[2,20]$} \\
GPRS-2G GSM & $64 \mathrm{Kbps}$ & $900 \mathrm{MHz}-1800 \mathrm{MHz}$ & $100 \mathrm{~m}$ & {$[21]$} \\
3G & $14.4 \mathrm{Kbps}-2 \mathrm{Mbps}$ & $1.6-2 \mathrm{GHz}$ & $100 \mathrm{~m}$ & {$[21]$} \\
4G-LTE & $100 \mathrm{Mbps}-1 \mathrm{Gps}$ & $2-8 \mathrm{GHz}$ & $100 \mathrm{~m}$ & {$[14]$} \\
LoRa & $0.3-50 \mathrm{Kbps}$ & $433,868,780,915 \mathrm{MHz}$ & $2-5 \mathrm{~km}$ & {$[1,22]$} \\
Bluetooth LE & $1 \mathrm{Mbps}$ & $2.4 \mathrm{GHz}-2.485 \mathrm{GHz}$ & $>100 \mathrm{~m}$ & {$[5]$} \\
RFID & $400 \mathrm{Kbps}$ & $125 \mathrm{KHz}-915 \mathrm{MHz}$ & $3 \mathrm{~m}$ & {$[23]$} \\
\hline
\end{tabular}

\subsubsection{Precision Agriculture Routing Protocols}

Data routing algorithms play an important role in WSNs by establishing the path of communication for data exchange between sensor nodes and base stations on a network. A variety of routing techniques have been proposed until now, aiming to achieve higher performance with minimal power consumption. IoT and WSN routing protocols can be categorized according to network structure and the way information will be disseminated through the network. A routing protocol can belong to more than one category, aiming to satisfy as many performance metrics as possible.

According to the way by which routing decisions are made proactive and reactive routing techniques can be used. Proactive routing (or table-driven) supports the periodic renewal and updating of the routes and destinations that are formed between the nodes throughout the network. On the other hand, reactive routing (or on demand) includes discovering routes on demand based on the transmission of route request packets. The downside to reactive protocols is their latency, since transmissions over unknown or expired routes face delays, for which either the application or the routing protocol must account by buffering or dropping data. 
Furthermore, according to WSN structure, routing algorithms can depend on neighbor nodes to broadcast the collected information. Another popular technique is based on dividing the network into clusters. Each cluster depends on a cluster head node to manage the routing of information between other clusters or base stations. Hierarchical routing is the most popular routing method in smart farming monitoring systems and soil parameter monitoring [23,24]. Multi-path routing protocols can also be used to implement a smart farm monitoring system to balance the data transfer load and conserved energy. Table 4 presents characteristic examples of routing protocols adopted in smart farming systems according to literature.

Table 4. Smart farming routing protocols.

\begin{tabular}{|c|c|c|}
\hline Routing Protocols & Category & Features \\
\hline $\begin{array}{l}\text { Destination-Sequenced } \\
\text { Distance Vector (DSVD) }\end{array}$ & Proactive & $\begin{array}{l}\text { Route availability to all network destinations with } \\
\text { minimal delay. }\end{array}$ \\
\hline $\begin{array}{l}\text { Link Estimation Parent } \\
\text { Selection (LEPS) }\end{array}$ & Proactive & $\begin{array}{l}\text { A map of the network is kept regarding the } \\
\text { interconnection of nodes. }\end{array}$ \\
\hline $\begin{array}{l}\text { Tiny Lightweight UNderlay } \\
\text { Ad-hoc Routing (TinyLunar) }\end{array}$ & Reactive & $\begin{array}{l}\text { Provided interfaces help to form route } \\
\text { characteristics. }\end{array}$ \\
\hline $\begin{array}{l}\text { Ad-hoc On-Demand } \\
\text { Distance Vector(AODV) }\end{array}$ & Reactive & $\begin{array}{l}\text { Used in ZigBee communication protocol for } \\
\text { interconnection of sensor nodes. }\end{array}$ \\
\hline $\begin{array}{l}\text { Dynamic Source } \\
\text { Routing(DSR) }\end{array}$ & Reactive & $\begin{array}{l}\text { A route on demand is formed when a transmission } \\
\text { node requests it. }\end{array}$ \\
\hline $\begin{array}{l}\text { Optimized Link State } \\
\text { Routing Protocol (OLSR) }\end{array}$ & Flat Routing & $\begin{array}{l}\text { Information about the status of the nodes is used to } \\
\text { select the appropriate path for packet forwarding. }\end{array}$ \\
\hline ProtoSense & Flat routing & $\begin{array}{l}\text { Reliable retransmission of information using } \\
\text { confirmation messages. }\end{array}$ \\
\hline $\begin{array}{c}\text { Periodic Threshold-Sensitive } \\
\text { Energy-Efficient Sensor } \\
\text { Network (APTEEN) }\end{array}$ & $\begin{array}{l}\text { Hierarchical } \\
\text { Routing }\end{array}$ & $\begin{array}{l}\text { It takes into account energy saving and network } \\
\text { lifetime [24]. }\end{array}$ \\
\hline $\begin{array}{l}\text { Location Routing Algorithm } \\
\text { with Cluster-Based Flooding } \\
\text { (LORA-CBF) [25] }\end{array}$ & $\begin{array}{l}\text { Location-based } \\
\text { routing }\end{array}$ & $\begin{array}{l}\text { It uses the flood method in a hierarchical network } \\
\text { structure to route data packets. }\end{array}$ \\
\hline
\end{tabular}

\subsection{The Encapsulation Layer}

Most of the current agro-environmental monitoring applications are based on machine to machine (M2M) communication support regarding real-time data transmission. There is a variety of communication patterns to be used so that the terminal can receive the information necessary to monitor the production. WSN technologies, enhanced by the IoT paradigm, enable smart sensor communication and connection to the IPv6-based internet, by addressing the agricultural sensors with IPv6 long addresses that can fit in lightweight IoT data link frames. IoT can immensely improve the autonomous capabilities of resourced-constrained nodes in a Low-power and Lossy Network (LLN). An agricultural WSN is a LLN. In such a network, some of the nodes may have a direct Internet connection to send and receive messages from the Internet. However, other nodes from the same network, may lack that kind of ability due to hardware limitations, and require the use of the Internet-connected nodes to access external services. Data exchange in a local context is also possible without the necessity of transmitting data to the Internet. The task of discovering the routes and allowing data messages to be transmitted among agricultural sensor nodes is performed by the routing protocol. Due to this fact, in such networks, network performance is strongly related to how the routing protocols use the limited hardware resources of the network device.

- The 6LoWPAN $[7,26]$ is the most popular network encapsulation protocol for precision agriculture applications. It refers to the transmission of IPv6 protocol packets over Low-Power Wireless 
Personal Area Networks. In a smart farming monitoring system, it is used by sensor devices that are compatible with the IEEE802.15.4 standard for WSNs. 6LoWPAN efficiently encapsulates IPv6 long headers in IEEE 802.15.4 small data frames for information exchange between sensor nodes. The advantages of this protocol are that it uses a special header compression method and a fragmentation process to reduce the transmission overhead [10].

- The IPv6 over LoRa [27] implementation enables the transmission of IPv6 protocol packets over LoRa links. If LoRaWAN is chosen for sensor node communication in a smart farming monitoring system, LoRa is the MAC protocol responsible for establishing communication between the LoRa gateway and the LoRa sensor end devices. In an agriculture monitoring system, the IPv6 adaptation enables the deployment of the IoT paradigm as a separate architectural layer. The basic aim of this layer is to manage header compression and packet fragmentation to deal with the requirements of LoRa modulation in the physical layer.

- The IPv6 over 802.11ah [10] or Wi-Fi-ah (HaLow) is a low-power/low-rate protocol able to support numerous sensor node devices on a single base station. This technology can be used for precision agriculture by enabling wireless base stations in the field to transmit data while also being energy conservative. Wi-Fi-ah (HaLow) uses special characteristics of the 6LoWPAN technology for effective transmission of IPv6 protocol packets over IEEE 802.11ah wireless networks.

- $\quad$ RPL (IPv6 Routing over Low Power and Lossy Networks) [26] is the most popular IoT routing protocol based on the distance vector routing technique. It is a proactive protocol that constructs a specific graph able to direct all traffic towards the sink node. RPL is the ideal routing protocol for agricultural LLNs, since it can quickly create network routes between sensor nodes in the field, share routing knowledge and adapt the topology in an efficient way. It is also efficient for multi-hop, many-to-one and one-to-one communication.

- LOADng-IoT is another IoT routing protocol, proposed in [28] as an enhancement for reactive protocol LOADng, which is considered to be the best current solution for LLNs. LOADng-IoT is able to boost the process of route discovery, reduce the overhead of control messages, and improve the network's quality-of-service(QoS). In a smart farm monitoring system, this protocol will allow sensor nodes without an Internet connection to forward their data packets to external Internet services with much greater reliability and lower latency.

\subsection{The Middleware Layer}

The Middleware Layer is responsible for establishing and controlling the association between IoT sensor devices. More specifically, the services of this layer include smart device management by the use of different transport protocols to forward the generated data from the involved IoT devices. Most of these transport standards use the Transmission Control Protocol (TCP) or the User Datagram Protocol (UDP) for forwarding information. There is a wide variety of data protocols [29] that belong to this layer towards developing an efficient managing platform for smart field devices. However, choosing the most appropriate device management technology depends on software and hardware specifications, network and technology architecture and communication standards being used in the according system to achieve maximum and efficient performance. In the view of using different kind of smart entities in an agriculture monitoring system, the services of this architectural layer facilitate the desired interoperability of the technologies introduced. All collected data will be stored on a remote server and transmitted to the client machine through Application Programming Interfaces (APIs) that provide web services for third-party users. APIs enable device communication in an application level for different kinds of purposes and are based on different architectures to transfer data from the server to the client.

- $\quad$ The MQTT-SN (Message Queuing Telemetry Transport For Sensor Networks) is a messaging protocol that facilitates device data collection and communication with servers using brokers. A broker is a network entity which arranges transactions between other network entities. By using the MQTT protocol, a precision agriculture monitoring system can enable smart sensor devices to 
publish messages to a broker and/or subscribe to a broker in order to receive certain messages. The exchanged messages will be organized by topics that act as a system for dispatching messages to subscribers.

- The CoAP (Constrained Application Protocol) is another popular protocol for IoT device data management. CoAP is based on a request/response pattern of communication allowing constrained devices to have web service functionalities. It is an HTTP-like web transfer protocol with the ability to extend the Representational State Transfer (REST) architecture to Low-Power Wireless Personal Area Networks (LoWPANs). REST is an architectural style for providing standards between computer systems on the web, while distinguishing the concerns of client and server.

- The XMPP-IoT (Extensible Messaging and Presence Protocol) is an open technology for real-time communication based on XML messages between connected devices and the available server. XMPP can efficiently power instant messaging, collaboration and content syndication in a smart farm monitoring system between all network entities.

- Device and asset management in a precision agriculture monitoring system can also be implemented using the Mihini [30] software. Mihini is an open-source project by Eclipse Technology that enables communication between an M2M server and the applications running on an embedded gateway. M3DA is the protocol used for the transport of M2M data. M3DA can allow user applications to exchange typed data/commands back and forth with an M2M server, in a way that optimizes the use of bandwidth.

- $\quad$ The OMA SpecWorks's Lightweight M2M [31] is another device management protocol for M2M or IoT devices. It can be used in a smart farming information system to efficiently transfer service data from the network to resource-constrained devices. In contrast to traditional M2M standards in which a device usually needs to keep up multiple stacks of technologies, protocols and security services, the LwM2M scheme allows the existence of one stack of technology for device management, not only on the level of the device itself, but also on the application level. In addition, LwM2M is based on protocol and security standards from the Internet Engineering Task Force (IETF).

- The ONEM2M [32] technical specification standards are an upcoming solution for device and asset management in precision agriculture. ONEM2M is a middleware IoT platform that provides functions and APIs for different service domains dealing with interoperability challenges. There are commercial and open-source implementations of this technology.

- A popular queuing protocol for enabling server connection in IoT is the AMQP (Advanced Message Queuing Protocol). This open standard protocol can facilitate message orientation, queuing, routing, reliability and security in precision agriculture applications.

- Last but not least, the DDS (Data-Distribution Service) [33] is the first open international M2M standard directly addressing publish-subscribe communications for real-time and embedded systems. This protocol has the advantage of providing fast data, event, and command exchange among the IoT sensor nodes in a precision agriculture monitoring system.

\subsection{The Configuration Layer}

The Configuration Layer deals with protocols running on top of the IoT monitoring platform allowing the exchange of data specified by its context regarding what is described, what was measured, when, where, by what, the time of validity, ownership, and others. The main objective of this layer is to curate, harmonize and aggregate the collected raw data, so that it can be published as context information, or supplied to upstream data processing algorithms or analytics. It is also capable of sending operational commands to the Middleware Layer. Context information is a term widely used in IoT research and characterizes data that may come from existing systems, users, through mobile applications, IoT smart devices, agricultural machinery or public geo-services. The Configuration layer uses an IoT broker to guarantee a common interpretation of information produced by heterogeneous data sources that typically employ different data formats and ontologies, and therefore are unable to 
directly share information among them. An IoT broker enables the forwarding of lower-level device information to higher-level Thing Information.

- Regarding precision agriculture applications, a popular context broker is implemented by the FIWARE NGSI technology, named Orion Context Broker. FIWARE is a framework of open-source platform components towards the deployment of the IoT paradigm. FIWARE NGSI is the FIWARE version of the OMA NGSI, an API based on HTTP that enables the integration of components and provides the basis for the interoperability and portability of IoT-enabled Smart Agriculture applications [34]. NGSI is an information model developed by OMA SpecWorks to manage context information with a meta-model based on entities, attributes and metadata. This protocol manages data concerning context entities, such as the lifetime and quality of information.

- The implementation of a smart farming monitoring system is greatly depended upon geo-services, location detection tools and mapping technologies. In such systems, the exchange of geographical information should be effortlessly accomplished between the involved network entities across the web. The Open Geospatial Consortium-Web Feature Service (OGC-WFS) [35] constitutes a desirable asset in formulating geographic information and offering direct fine-grained access at feature property level of the data to IoT sensor nodes in precision agriculture applications. OGC offers various standards that can ease the way location data is exchanged and stored in a smart farming system that is based on drone monitoring. Furthermore, the OpenGIS Web Map Service Interface Standard (WMS) [35] can be efficiently used by UAVs, since it provides a simple HTTP interface for requesting geo-registered map images from one or more distributed geospatial databases.

\section{The Management Layer}

In a smart farming monitoring system, the basic component of intelligence is considered to be the study and filtering of the collected data. The use of EO techniques enable the advance of cultivation procedures and increase productivity by providing the base layer for spatial information analysis and monitoring of agricultural activities. A large percentage of smart agriculture applications are based on simulators, commercial programs and specific programming languages for implementing and controlling the data system. The Management layer uses modern software tools to efficiently satisfy multiple tasks, presented in Table 5.

Information management is deployed so the farmer can consult, record and modify the information collected by the sensors in tables, statistical graphs and interactive maps. In addition, it can download daily, monthly and annual reports of historical data. However, the farmer can mainly see the current data of the monitored variables of one or all the WSN nodes and consult the history. The interaction with the network and services layer is achieved using an intermediate layer of management logic [36]. WSN data will be stored in an online database [37].

The system also enables Big Data analytics in agriculture monitoring by using tools such as the Apache Hadoop software and various Big Data hardware platforms [38] as possible resources. Big Data refers to information assets characterized by such a high volume, velocity and variety able to require specific technology and analytical methods for its transformation into value [39]. In precision agriculture smart applications, the collected data are recorded in a specific format, to discover patterns, correct errors, eliminate duplicate and inconsistencies and to solve noise problems. Big Data technologies are playing an essential role in modern farming systems, since predictive insights are provided regarding optimizing the quality of the crop, minimizing environmental impact, reducing costs, increasing profit and generally optimizing production efficiency. In addition, Big Data enable the management of real-time operational decisions and the redesign of business processes for advancing the food supply chain.

Furthermore, the proposed architectural scheme promotes various data processing techniques that upgrade the impact of EO technologies in agriculture. EO by satellite leads to the acquisition of regular or spatially continuous data regarding large areas. It provides essential information on the functioning 
of fields and on the causes of environmental change. UAVs enable EO by using photogrammetry techniques to extract three-dimensional digital surface models of the field, as well as orthophotographs. One popular algorithm for this purpose is Structure from Motion (SfM) [40]. An orthophotograph can be used to create a realistic map of the fields by measuring true distances and providing to the farmer a clearer helpful view from the sky. Based on photographs, digital image processing tools, such as Pix4Dmapper can calculate various vegetation indices that can lead to conclusions, either on each photograph individually or after the production of orthophotos regarding the crops' state. Vegetation indices are mathematical quantitative combinations of the absorption and scattering of plant in different bands of the electromagnetic spectrum [41]. Calculating vegetation indices will help to identify useful crop characteristics concerning important biological and physical parameters of the vegetation. The best-known vegetation index is the Normalized Difference Vegetation Index (NDVI), which is the evolution of the vegetation index ratio and is calculated by the visible and near infrared light reflected from the vegetation.

Last but not least, the smart agriculture monitoring system also performs data mining processes based on tools such as the Apache Mahout Framework so as to identify and discover hidden patterns in the collected data, once they are processed, in the form of reviews. In addition, Machine Learning techniques will be used in the smart monitoring platform in order to estimate the extracted parameters of the crops' growth rate and also help to identify objects or animals trough the collected images by using Object-Based Image Analysis (OBIA) [42]. Moreover, new machine learning models-algorithms focused on data classification $[43,44]$ — can be used to minimize the size of redundant data and fasten the analysis.

Table 5. Smart farming monitoring system services.

\begin{tabular}{|c|c|c|}
\hline Service Type & Tools & Description \\
\hline \multirow{2}{*}{$\begin{array}{l}\text { Information } \\
\text { management }\end{array}$} & Database & $\begin{array}{l}\text { The central server database for storing and maintaining the sensor } \\
\text { collected data, management commands and application user } \\
\text { information. }\end{array}$ \\
\hline & Management logic & $\begin{array}{l}\text { The process of managing the systems units, organizing and } \\
\text { displaying the evaluated data into a user-friendly way. }\end{array}$ \\
\hline \multirow{2}{*}{$\begin{array}{l}\text { Big Data } \\
\text { analytics }\end{array}$} & $\begin{array}{l}\text { Apache Hadoop } \\
\text { Framework }\end{array}$ & $\begin{array}{l}\text { Complex process of examining large and varied data sets with an } \\
\text { intention to uncover meaningful and useful information that can } \\
\text { help in deriving conclusion and take decisions. }\end{array}$ \\
\hline & $\begin{array}{l}\text { Big Data hardware } \\
\text { platforms }\end{array}$ & $\begin{array}{l}\text { The use of different hardware platforms for Big Data } \\
\text { analytics according to the available hardware, scale-ability and } \\
\text { performance characteristics of each platform. }\end{array}$ \\
\hline \multirow{3}{*}{$\begin{array}{l}\text { Data and Image } \\
\text { processing }\end{array}$} & $\begin{array}{l}\text { Digital Image } \\
\text { processing }\end{array}$ & Vegetation Indexes calculation \\
\hline & $\begin{array}{l}\text { Photogrammetry } \\
\text { techniques }\end{array}$ & $\begin{array}{l}\text { Extracting three-dimensional digital surface or terrain models of } \\
\text { the field and orthophotographs. }\end{array}$ \\
\hline & $\begin{array}{l}\text { Machine learning } \\
\text { classification } \\
\text { algorithms }\end{array}$ & $\begin{array}{l}\text { Classification of data to decrease the size of redundant } \\
\text { information and identify objects or animals. }\end{array}$ \\
\hline \multirow{2}{*}{ Data mining } & $\begin{array}{l}\text { Apache Mahout } \\
\text { Framework }\end{array}$ & $\begin{array}{l}\text { Systematic and sequential process of identifying hidden patterns } \\
\text { and information in a large dataset. }\end{array}$ \\
\hline & $\begin{array}{l}\text { Object-Based Image } \\
\text { Analysis }\end{array}$ & Identify objects or animals through the collected images \\
\hline
\end{tabular}

Furthermore, all these services are hosted in the cloud to be able to access them remotely from any geographical location. 


\section{The Application Layer}

Based on the proposed precision agriculture monitoring system architecture, the farmer can interact with the IoT applications of the system to remotely manage the cultivation process. Such applications may concern any aspect of the agricultural field ranging from planting and irrigation processes to plant protection and harvesting methods [45]. The applications that can be adopted may involve the fertilizer application, the weed mapping, the spraying process, the irrigation of the field and the alert system.

The Variable Rate Fertilizer (VRF) application has as a target to optimize the usage of nutrients by defining the amount of fertilizer applied based on the health of the plant. Variable rate fertilizer in precision agriculture is an area of technology that focuses on the automated application of fertilizer to a given landscape. The way in which the materials are applied is based on data that is collected by sensors, maps, and GPS. VRF applications bring several benefits related to savings on fertilizers and chemicals, potential yield increase and environmental protection. In the same context is the Variable Spraying application. These types of applications implement controllers that turn the herbicide sprayers on and off. Usually variable spraying applications take into consideration information coming from the weed mapping tools such as the weed locations. In that case the appropriate volume of herbicide is estimated and applied in the field based on the weed intensity.

The Weed Mapping application focuses on the visualization of the weed occurrences within a certain crop field with the help of mappings. The GPS receiver with an aerial vehicle generates maps which show the weed occurrences. These weed maps can be combined with fertilizer maps and yield maps. The IoT-based irrigation system use a micro-controller that serves as information gateway receiving real-time information from soil moisture and temperature sensors placed on the fields. Generally, a moisture/temperature threshold is specified based on which the micro-controller automatically switches on the water pump. The micro-controller also has servo motors to ensure that the area is uniformly irrigated. The entire system can be managed remotely by the end-user through the dedicated application.

Alert/ notification applications are also very popular in IoT-based precision agriculture. Producers and agriculture companies implement IoT solutions for instantly tracking their crop fields. In this case, the data coming from IoT devices is processed and transformed into knowledge properly visualized for offering information regarding the health of the vegetation and the soil, the behavior patterns of the plants, detect signs of disease on time, identify insects and harmful animals and instantly alert producers about potential difficulties. This type of applications serves for storing and analyzing data, providing producers with relevant recommendations.

The aforementioned applications aim at the efficient field and crop management to:

- increase production efficiency

- improve product quality

- provide more efficient use of chemicals in cultivation

- manage pesticide amounts

- reduce energy consumption

- $\quad$ protect the soil

- control water consumption and underground water amounts

The IoT-based agriculture applications can be implemented for an Android or Windows smart-phone, a tablet or as a web application. The applications of IoT-based smart farming apart from conventional, large farming operations, targets also other growing or common trends in agricultural such as organic farming, family farming (complex or small spaces, particular cattle and/or cultures, preservation of particular or high quality varieties etc.), and enhance highly transparent farming. Our precision agriculture monitoring system can also benefit the dry farming technique that encompass specific agricultural techniques for the non-irrigated cultivation of crops. Furthermore, greenhouses can use our architectural model to intelligently monitor as well as control the climate, eliminating the need for manual intervention. 


\section{Energy-Saving Techniques and Security Mechanisms}

\subsection{Energy-Saving Technologies}

In precision farming applications sensor nodes are usually powered by low-energy batteries that are difficult or impossible to recharge or replace. This is considered to be a major disadvantage to maintain a real-time monitoring system. Energy-saving techniques is vital to maintain the system's efficiency in smart farming. This kind of techniques can provide battery life extension by reducing the amount of communication between the nodes and the base station, while minimizing the redundant data in the network. Energy preservation techniques for precision agriculture systems are presented as a separate architectural level covering the sensing and networking procedures of smart farming.

In the Sensor layer, the proposed energy-saving approach is an on/off process which is based on the selection of a subset of nodes that will remain active for a certain period of time, while others remain inactive. Following this assumption, SWORD (sleep/wake on redundant data) is an energy preserving scheme that can be used to collect data on soil moisture [19]. The SWORD algorithm performs data control by removing redundant data so as to minimize energy consumption and increase the life of sensor nodes in the network.

In the Network layer, data transmissions and receptions can also be scheduled based on the sleep/awake periods of sensor nodes at predetermined intervals. For this purpose, $A^{2} S$, an automated agricultural precision tracking system can be used [46]. Based on this energy-saving technology, whenever the sensing period is set by the application server, the sink node keeps the schedule and it spreads the sleep order message over its network every sensing period. Each time a node receives the sleep message, it sets the sleep timer's end time to the value of the duration field included in the message. When the meter time ends, the node detects the environment and battery voltage level and sends the data to the source. Then, he expects the next sleep request message.

Moreover, another energy-saving scheduling technique that can be deployed in the Network layer involves the use of unmanned flying vehicles in an agricultural crop monitoring system. Based on this scheme, the node on the unmanned flying vehicle wakes the ground nodes to retrieve the measured data. To perform this function, a coded radio signal is sent via a transmitter to the ground nodes. The nodes are in an inactive state, except for a small receiver waiting to receive the trigger signal.

Furthermore, taking advantage of APTEEN hierarchical routing protocol, a time division multiple access technique can be implemented as a scheduling method. Based on this technique, messages are sent to put some nodes in sleep mode so as to avoid packet collisions between sensor nodes belonging to different clusters. In addition, carrier sense multiple access technique is another alternative method, which is equally effective for avoiding collisions.

\subsection{Security Mechanisms}

Precision agriculture monitoring systems involve the exchange of sensitive information regarding the cultivation process, the state of crops and personal data of authorized staff. It is of great importance for such systems to be protected against cyber-attacks. An unauthorized entry of a malicious individual in the system may cause great damage to the cultivation process or even acquire the farmers personal information [47]. In the pursuit of safeguarding a smart farm monitoring system the confidentiality of data should be met. In addition, it is vital to ensure the reliability of the data and the ability to confirm that a message has not been tampered with, altered or changed while on the network. Also, the services of resources offered by the network, or by a single sensor node must be available whenever required and finally to be able to identify the origin of a message received. Basic security mechanisms are already deployed by the combined IoT technologies in every architectural level of the proposed smart monitoring system. However, there are more ways to increase the level of security in each layer separately.

One popular way of providing physical layer security in WSN and IoT systems is cryptography. Physical layer security refers to the inner security capabilities of the Sensor layer regarding the 
randomness of wireless channels, the signal-to-noise ratio gaps or intended jamming. There are three kinds of cryptographic methods used in WSNs, the symmetric and asymmetric ones and hash functions. Hash functions are special mathematical functions which map a given input to a certain output with a fixed size. Well-known cryptographically secure hashes are the Secure Hash Algorithm-2 (SHA-2) and Advanced Encryption Standard (AES) algorithm. In symmetric cryptographic techniques, a single shared key is used between the two communicating nodes both for encryption and decryption. This key stays known only to the nodes of the network. On the other hand, in asymmetric cryptography, a private key can be used to decrypt and sign data. A public key is also used to encrypt and verify data. The private key needs to be kept confidential while the public key can be published freely. Asymmetric cryptographic techniques may use the RSA (Rivest-Shamir-Adleman) algorithm, the ECC (Elliptical Curve Cryptography) algorithm or the pairing technique. Popular frameworks of symmetric and asymmetric technique can be found in [48].

Regarding the Link layer of our proposed architecture security mechanisms focus against the interception, modification and fabrication of the exchanged data. Attack detection mechanisms can be applied, such as the misbehavior-aware threshold detection scheme for LLNs proposed in [49]. In addition, secure routing protocols may be used such as SAR(Secure aware routing protocol), which is based on on-demand protocol such as AODV or DSR and DPRAODV (Detection, Prevention and ReactiveAODV) [50].

Middleware security mechanisms deal with unauthorized modifications that occur due to transmission errors (accidental) and require the use of digital signatures. Digital signature schemes are cryptographic schemes that include key generation algorithms, signing algorithms and signature verifying algorithms. A digital signature is an authentication mechanism that enables the creator of the message to attach a code that acts as a signature. The Digital Signature Algorithm (DSA), developed by the National Institute of Standards and Technology, is one of many examples of a signing algorithm.

Access control is an important building block for the overall security of a precision agriculture monitoring system. The IoT requires access control models that apply authorization policies across a multitude of smart sensors. In a smart farm monitoring platform, authentication is required to prevent authorized users from accessing resources in an unauthorized manner. For instance, a worker in the fields is a legitimate user but does not have the access rights as the main administrator farmer. Most importantly, access control prevents illegitimate users from gaining access to resources. The Attribute-Based Access Control (ABAC) model is a technique that assigns attributes to each entity in the system. The attributes may refer to either a user or a resource and are defined as properties of every entity to enable authentication. Another paradigm able to provide solid communication in the application layer is the Role-Based Access Control model (RBAC) where a user is assigned as administrator or ordinary user that predetermines access rights policies. In the Application layer, each user can be authenticated by the use of passwords or a smart key card [51].

\section{Use Case Study: The DIAS Architecture}

The DIAS research project stands for Drone Innovation in saffron Agriculture Surveillance. It is co-funded by the European Union and Greek national funds through the Operational Program Competitiveness, Entrepreneurship, and Innovation. University of Western Macedonia (UOWM) in cooperation with Kozani Saffron Producers Cooperative (KSPC) aims at developing an integrated automated surveillance system for saffron cultivation. This $24 \mathrm{~h}$ real-time saffron cultivation surveillance system is relied on signal and image collecting and processing, which is derived from advanced surveillance, risk identification and early warning systems, based on integrated sensor networks, and aerial unmanned vehicles.

\subsection{Saffron Cultivation}

The saffron cultivation is considered to be of extreme importance for the Western Macedonia citizens, due to the activity over saffron cultivation by a large number of citizens, the limited farming 
geography, the unique production process to plant the saffron seeds, and the unique production risk that this operation faces. The only kind of saffron that is systematically cultivated around the world, for at least ten centuries, is the edible saffron or as it known in scientific (botanical) terminology Crocus Sativus Linneaus.

The cultivation process includes harvesting the flowers, as presented in Figure 2, splitting the stigmas and stamens from the petals, drying and sorting of saffron. The picking of 1000 flowers is process of a duration around $45-55 \mathrm{~min}$, while additional 100 to $130 \mathrm{~min}$ are required for removing the stigmas for drying. In total, 370 to $470 \mathrm{~h}$ are required to produce $1 \mathrm{~kg}$ of dried saffron [52]. The flowers are picked exactly when they are fully bloomed, and the saffron strand or stigma is at its reddest. The harvesting process begins shortly after dawn to minimize the further sunlight exposure to the crops, since they may lose their color and even flavor. Saffron cultivation requires dry soil with specific levels of moisture, controlled irrigation and right amounts of nitrogen, phosphorus and potassium. Cultivation is commonly disturbed by mice, moles and rats wrecking the stems. Furthermore, fungi may cause specific diseases at the early stages of saffron growth.

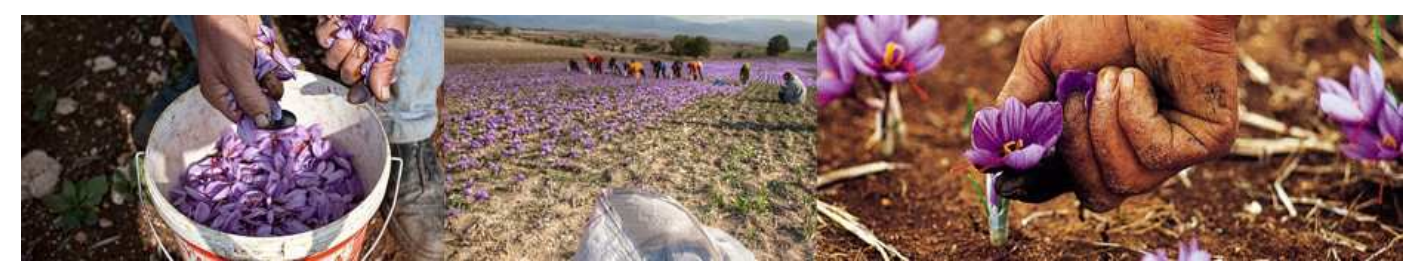

Figure 2. Saffron harvesting.

The DIAS platform aims to improve the production process, by offering the ability of immediate interference in case of animal, disease or wild weeds detection. The farmer will be able to monitor the fields in real time and getting alerts in case of unwanted incidents regarding the state of the plants during all cultivation period, while raising profits. To satisfy these goals, the monitoring process will be aligned specifically with the farmers concerns and actions during each month of cultivation as presented in Figure 3.

\begin{tabular}{|c|c|c|c|c|c|c|c|c|c|c|c|c|}
\hline $\begin{array}{l}\text { Necessary actions I } \\
\text { Month of cultivation }\end{array}$ & January & February & March & April & May & June & July & August & September & October & November & December \\
\hline \multicolumn{13}{|l|}{ Irrigation } \\
\hline \multicolumn{13}{|l|}{ Check for Animals } \\
\hline \multicolumn{13}{|l|}{ Fertilizer } \\
\hline \multicolumn{13}{|l|}{ Check for WildWeeds } \\
\hline \multirow{2}{*}{\multicolumn{13}{|c|}{$\begin{array}{c}\text { Milling } \\
\text { Bulb extraction } \\
\end{array}$}} \\
\hline & & & & & & & & & & & & \\
\hline \multicolumn{13}{|l|}{ Cleaning } \\
\hline \multicolumn{13}{|l|}{ Replanting } \\
\hline Harvest & & & & & & & & & & & & \\
\hline
\end{tabular}

Figure 3. Saffron cultivation yearly stages.

\subsection{DIAS Architecture}

The DIAS platform follows the architectural design of the proposed remote sensing monitoring system. As presented in Figure 4 the DIAS system is consisted of seven architectural layers. In each layer the most suitable aforementioned technologies are used towards achieving the overall system's efficient performance and reliable operations. The saffron cultivation is a quite demanding and delicate process requiring the deployment of tailor-based technologies and continuous crop monitoring to avoid animal and disease interventions.

In the Sensor layer, quadcopter UAVs with thermal, hyperspectral and RGB cameras are being used in cooperation with multiple wireless nodes equipped with various sensors for data collection regarding the crops. Saffron cultivation monitoring requires the study of small sized crops and the notice of minimal crop state variations during each month. To select the desired information UAVs must fly in a certain distance and place for a specific period of time and by using different 
position angles. Quadcopter UAVs is considered to be the best choice for saffron monitoring since they have rotary wings, which provide them with the ability of maneuverability, while also flying in low speed. Regarding RGB cameras, they are ideal since saffron cultivation requires the capture of data-images in different weather conditions avoiding inadequate or excessive exposure of the image. Using multispectral or hyperspectral sensors, UAVs can also obtain information related to spectral absorption and reflection of the crops in several bands. This quite useful since the provided information can then be used to calculate vegetation indices and monitor the state of saffron crops based on them. On the other hand, thermal imaging detects specific levels of radiation and translates it into a grayscale image, using brighter and darker shades of heat representation. This ability is also essential in saffron cultivation since the fields are regularly trampled by animals that are not being detected on time in before they cause any damage. The DIAS monitoring system uses different ground and on-leaf sensors to collect useful data for improving the cultivation process. More specifically, environmental humidity and temperature is evaluated since saffron requires specific conditions to prosper. Additionally, the levels of luminosity, leaf-wetness, soil moisture, $\mathrm{pH}$ and wind speed are also of great importance. Furthermore, the amounts of nitrogen, phosphorus and potassium in the soil can have a severe impact on the plant's growth, as well as the moisture levels in the soil. The farmer will be able to predict production loss efficiently due to a round knowledge of the saffron fields condition and even intervene with possible treatments to change its course for the better.

Regarding the Link and Encapsulation layer, sensor data exchange in the DIAS platform is enabled by the LoRaWAN technology and the IPv6 protocol. LoRa technology offers several advantages. The LoRa physical layer uses ISM bands 868 and $915 \mathrm{MHz}$ - frequencies that are free to use anywhere in the world. In addition, LoRa devices consume very little power making it ideal for battery-powered devices and it can also transmit and receive data for up to $15 \mathrm{~km}$ in suburban areas and $5 \mathrm{~km}$ in urban areas. Another advantage of LoRa is its high network capacity. In LoRaWAN, a single gateway can accommodate 1000 end-node devices. On the other hand, IPv6 offers large addressing space and has built-in support for network auto-configuration. In the DIAS platform, each sensor node is characterized by its own IP address and uses a power saving scheduling and routing protocol to transmit the collected data to the nearest LoRa Gateway. The information to be exchanged is encrypted before transmission. Each LoRa gateway forwards the information to the according network router for Internet access and data storage to the system' database. UAVs' data transmission and communication is enabled by the IEEE 802.11ah networking standard in cooperation with the IPv6 protocol, to forward the collected images to the network server as well.

In the Middleware layer, the DIAS platform will employ the MQTT-SN protocol. MQTT-SN enables the management of all DIAS networking devices through message exchanges coordinated by the FIWARE's context broker. It is essential to maintain the monitoring's system reliability towards achieving meaningful data collection. Based on this protocol, the exchanged messages can be organized by topics and importance regarding device management commands, transmission requests initiated by a higher layer and data acquisition. Sixteen number of message types are supported and transmitted in an asynchronous way of communication by the publish-subscribe model. TCP and UDP will also be used as transport protocols. MQTT is ideal for the DIAS system since it excels in smart sensor device communication on a wide area network, due to the publish/subscribe architecture with the broker in the middle. MQTT is also very useful if the bandwidth is limited to enhance network reliability and availability.

In the according Configuration layer, all saffron field collected data will be grouped and formulated to reach the information management unit. This layer facilitates the FIWARE NGSI API so to produce the desired information model of the acquired field data based on efficient context management. The FIWARE's Orion Context Broker has powerful features enhancing the capabilities and performance of the DIAS monitoring system such as enabling asynchronous application notifications. The basic operations of the current layer include enabling the publication, 
consumption, subscription and processing of all the information relevant to the crops and the cultivation process in the DIAS platform.

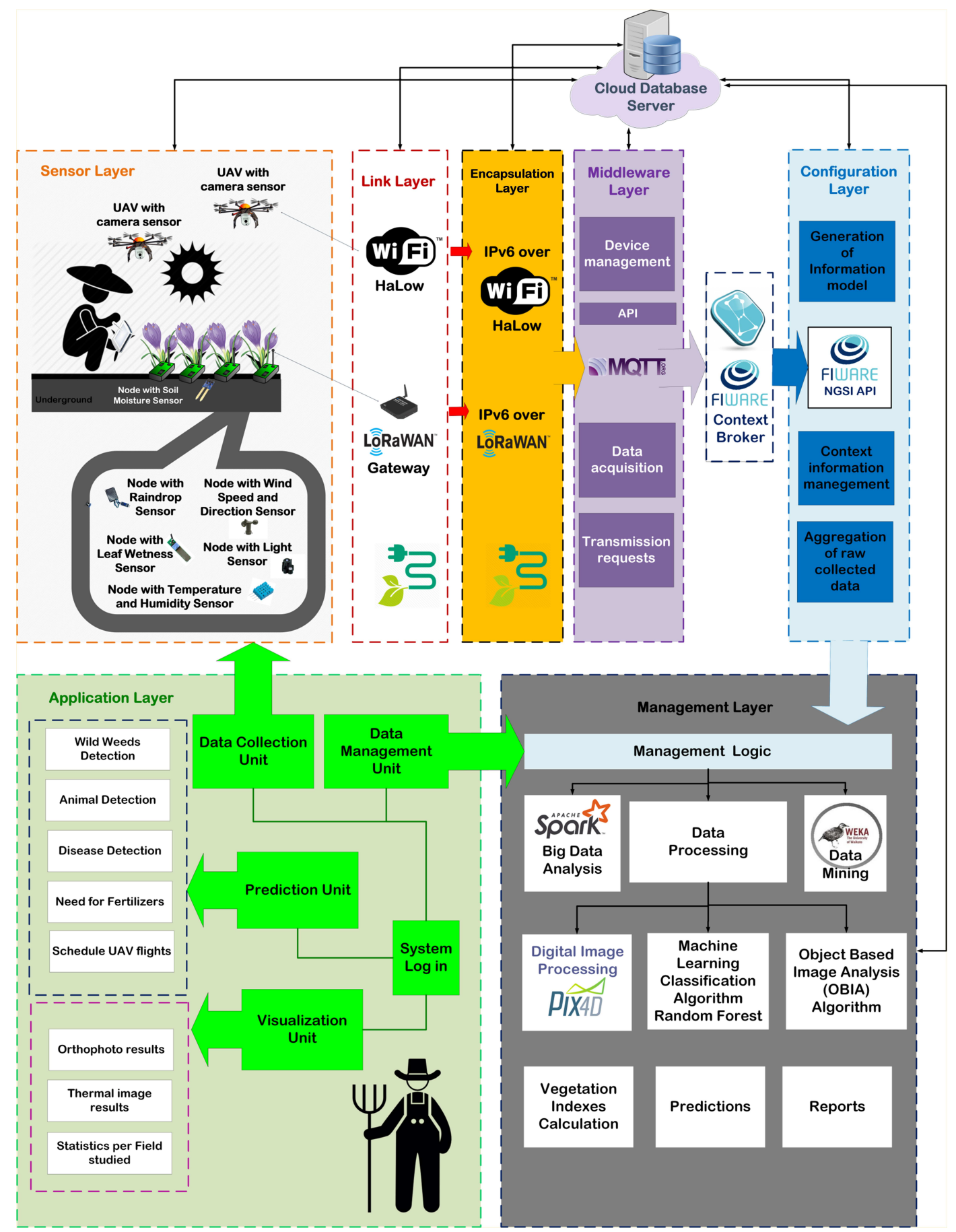

Figure 4. The DIAS architecture.

The Management layer constitutes the basic operation center of the DIAs platform. It stores, maintains, analyzes the collected data and produces predictions for the progress of the crops growth rate and statistics regarding they current state. According to the commands acquired from the user of the platform in the highest architectural layer, the information management logic initiates the according processes. Data processing includes the use of the Random Forest machine learning classification algorithm for direct extraction of conclusions regarding the growth and health of vegetation. This algorithm is directly applied on the data of the images acquired by the UAVs, evaluating the RGB 
colors and the intensity of each pixel. The Random Forest scheme performs very well with high dimensionality and can handle binary features, categorical features, and numerical features. This method is chosen since very little pre-processing is required and the data does not need to be rescaled or transformed. Digital image processing of geospatially corrected aerial images (orthophotographs) focuses on producing the according vegetation indexes for evaluating the state of saffron plants. Two kind of vegetation indexes will be calculated by the PiX4D image processing tool. Vegetation indexes derived from multispectral information, such as NDVI will enable the detection of unhealthy or sparse vegetation that reflects more visible light and less near infrared light, making it easy to monitor the growth and health of saffron crops. On the other hand, regarding the use of RGB-based Vegetation Indices, Excess Greenness Index $(E x G)$ is based on the assumption that saffron plants will display a clear high degree of greenness, while soil being the only background element. Finally, the Normalized Difference Index (NDI) will be also used since it can enable the separation of saffron plants from soil and residue background images, using only green and red channels. Spectral information can help significantly in assessing a lot biological and physical characteristics of the saffron crops. Any kind of wild weeds or animal detection based on thermal imaging in the fields initiates alerts for immediate intervention and is taken into consideration as a note in the cultivation calendar created by the platform for the farmer. For this purpose, the OBIA algorithm is also used to recognize weeds or discriminate species. Object-based image analysis is based on the comparison between sets of similar pixels called image objects in measures of spectral properties (i.e., color), size, shape, and texture, as well as context. The OBIA algorithm is chosen since it is more suited to landscape-scale analyses and can filter out meaningless information and assimilate other pieces of information into a single object. After processing, all the collected sensor data are categorized and evaluated in accordance with the desired vegetation index values, moisture levels and general plant state for each month during the cultivation process. For these tasks, the DIAS platform employs powerful and well-known tools, the Apache Spark Framework focusing on Big Data analytics and the WEKA (Waikato Environment) Framework specializing in data mining tasks. The evaluation results will point out the vulnerable spots in the field with helpful statistical graphs and suggest possible solutions to the farmer, such as the use of fertilizer.

In the Application Layer, each user can access the DIAS platform by using his/her own unique password. According to their authority and activities in the saffron field the users have different access rights in the DIAS platform. A user may be the administrator of the platform, a representative of the KSPC board, a farmer or a worker in the fields. For instance, a worker can only observe the evaluation results in order to aid the farmer in treating the plant growth, while the KSPC representative can add observation notes, can schedule and initiate sensor data collection in a specific field and also manage the flight plan of UAVs. On the other hand, the platform administrator is only one who can add new users and new saffron fields for cultivation to be observed in the system. Access in the DIAS platform is forbidden for non-authorized users. In the Application layer, the management of the four basic operational units of the DIAS system can be initiated. These are the Visualization Unit, the Data Management Unit, the Prediction Unit and the Data collection Unit. By entering the Data collection Unit, a user can initiate sensor data collection in a selected field or modify existing data in the platform. By entering the Data Management Unit, the user can evaluate orthophotographs and calculate vegetation indexes according to specific data in a specific saffron field with the help of Pix4DMapper tool. The Visualization Unit is related to the presentation of statistical results and graphs regarding each saffron field, while the Prediction Unit employs the aforementioned data mining tools to produce a sufficient evaluation of the collected data. All system information is stored in the central server database.

Based on the acquired and processed data, farmers can produce statistics regarding the progress in saffron production and avoid financial loss, while improving the quality of the crops and accomplishing a considerable rise in production. 


\subsection{Benefits and Costs}

Greece is the second largest saffron producing country, with an average output of 4 tons of p.a. during the last four decades, most of which is directed in export markets. The establishment of the 'Kozani Saffron Producers Cooperative' marked the beginning of a good decade for this product with production reaching its height record of more than 12 tons in 1982 [53]. Nevertheless, in the period following Greece's accession to the European Community, farmers began abandoning saffron cultivation. This fact resulted in a significant decrease in yield with an average yield of $800 \mathrm{~g}$ per stremma $\left(1 \mathrm{ha}=10\right.$ stremmas $\left.=1000 \mathrm{~m}^{2}\right)$. However, this trend seems to be reversed since 2010 and currently yields vary around 1 ton per stremma. Currently, Greek saffron price is about 1200 euros per $\mathrm{Kg}$. Nearly 5000 spots are needed to produce $100 \mathrm{~g}$ of red saffron. The average produce per acre is $6 \mathrm{~kg}$ of dried stigmas (red product). The lower production of the plant is on the first year of the plantation, while the highest on the third and the fourth.

In the year of 2015, a study in Greek saffron cultivation resulted that farm managers need to address the efficiency of input use and raise capital and labor productivities to maintain competitiveness [53]. The saffron quality is determined by the color, flavor and size of the stigmas. The DIAS platform aims to upgrade profit earnings by the adoption of new technologies, ensuring flower picking at appropriate time in a proper collection material at an appropriate age. Animal intrusions in the fields, causing a huge loss in production, will be prevented and crops diseases will be detected in time. The overall equipment cost is considered to be a safe investment for farmers since most of the technologies involved are open source and the initial cost of the related hardware and software can be shared across producers. Currently, the DIAS initiative is under construction and no experimental results are available to confirm the proposed objectives. However, in Table 6 we present the production rate of Greek Saffron as recorded in [53], while in Table 7 an average estimation is provided regarding the increase in cultivation operations by adopting the DIAS platform. These percentages are the estimations provided by two of the producers whose fields participate in the pilot study. The estimations show that on average the farmers calculate an increase of around $20 \%$ in production, this percentage is in accordance to other types of cultivations such as olives and potatoes [54].

The use of the DIAS project can offer a significant increase in saffron production. Nevertheless, since no pilots have been implemented yet in the project, the Return on Investment (ROI) is difficult to be estimated. An investment of time and money from the DIAS consortium is required to investigate how the data gathered from UAVs can connect to and affect other things that are happening on the fields. Saffron growers need that information to provide context and create a correlation regarding causes and effects. At that point ROI becomes significant promoting UAVs as a part of the desired solution and not as the solution itself. Furthermore, due to shared costs between project partners, ROI becomes a collective performance measure that can be estimated only when each member has acquired the according technological benefits. A complex profitability study is required to specify the precision service offerings, as presented in $[55,56]$. Each partner in the DIAS project owns and offers different assets in this endeavor, thus calculating the overall ROI of UAV usage from the side of farmers is not yet possible.

Table 6. Greek saffron cultivation economic estimation in one year.

\begin{tabular}{cc}
\hline Average production results & 2200 euros \\
\hline Average produced yield & $1.8 \mathrm{Kgs}$ \\
\hline Average labor cost of annual working hours & 145 euros per $1000 \mathrm{~m}^{2}$ \\
\hline Average cultivated land & $15,000 \mathrm{~m}^{2}$ \\
\hline
\end{tabular}


Table 7. Economic estimation for Greek saffron cultivation via using the DIAS platform in one year.

\begin{tabular}{cc}
\hline DIAS platform hardware and software equipment cost & 45,000 euros \\
\hline Average increase in production results in euros & $10 \%$ \\
\hline Average increase in produced yield in Kgs per $1000 \mathrm{~m}^{2}$ & $20 \%$ \\
\hline Average increase in saffron quality per $1000 \mathrm{~m}^{2}$ & $25 \%$ \\
\hline
\end{tabular}

\section{Challenges}

The implementation and maintenance of a monitoring system in precision agriculture faces several challenges. The greatest challenge in the sensor layer is for sensor nodes to achieve efficient and continuous operation for a long time in a natural environment, while taking into account the climate change and wildlife interventions. The battery life of sensor nodes is not considered satisfactory, and it is necessary to design and implement energy-saving protocols with the highest possible system performance among other precautions. In addition, depending on the type of application, the supported agricultural work and the implementation technologies, the problems that arise can be differentiated. For instance, the use of sensors and controllers from different manufacturers prevents communication between them and makes it more difficult to interconnect with other agricultural components. Also, the sensor inertia phenomenon was observed in a high-speed WSN due to non-steep changes in humidity and soil temperature.

In the network layer, the basic challenges regarding the operation of a crop monitoring system with WSN and IoT technologies include the limited computational capabilities of sensor nodes. The restricted memory of the nodes disables them to handle large amounts of communication data and cluster-based interconnection procedures. Due to this fact, long data queues are created in each node, leading to greater delay in transmissions. The same outcome can be triggered by the long communication distance of sensor nodes. One major issue that routing algorithms must deal with in such cases is the high level of energy consumption, which leads to a reduction in the overall viability of the network. In precision agriculture monitoring systems routing protocols should offer minimum delay, be able to provide efficient services in many sensor nodes, while taking into account the limited resources. They should also be capable of accepting all sorts of environments including severe and loss environments, while providing information security and privacy. Most routing protocols use some localization technique to obtain knowledge concerning their locations. The performance of the routing protocol is a function of network size and transmission media. Therefore, transmission media of good quality enhances the network performance directly.

However, in many cases the failure of such advanced monitoring system may be due to the geographic, cultural or socio-economic distance between system designers and the intended user community. Cost is an important limiting factor in the implementation of such systems. The cost depends to a great extent on the quality of the materials and the topology of the network.

\section{Conclusions}

This paper proposes the architectural components of a smart farming monitoring system, based on modern IoT communication technologies and WSN capabilities, in cooperation with energy-saving protocol schemes. A use case study based on the DIAS project is also presented, enhancing the reliability and contribution of our model. The IoT agricultural applications enable farmers to collect and analyze meaningful data. Large landowners and small farmers should welcome the potential of IoT market for agriculture by installing smart technologies to increase competitiveness and sustainability in their productions. The rapid growth of population forces farmers to meet the demand by implementing agricultural IoT solutions in a prosperous manner. In the future, an in-depth study will be carried out regarding the real-time performance of the proposed model in the DIAS project. 
Author Contributions: Conceptualization, A.T., P.S. and S.B.; Methodology, A.T., P.S. and S.B.; Writing-original draft, A.T.; Writing-review \& editing, P.S. and S.B.

Funding: This research was co-funded by the European Union and Greek national funds through the Operational Program Competitiveness, Entrepreneurship, and Innovation, grant number T1EDK-04873, project “Drone innovation in Saffron Agriculture," DIAS.

Conflicts of Interest: The authors declare no conflict of interest.

\section{References}

1. Guillermo, J.C.; García-Cedeño, A.; Rivas-Lalaleo, D.; Huerta, M.; Clotet, R. IoT Architecture Based on Wireless Sensor Network Applied to Agricultural Monitoring: A Case of Study of Cacao Crops in Ecuador. In Advances in Information and Communication Technologies for Adapting Agriculture to Climate Change II; Springer International Publishing: Cham, Switzerland, 2019; pp. 42-57.

2. Donzia, S.K.Y.; Kim, H.K.; Hwang, H.J. A Software Model for Precision Agriculture Framework Based on Smart Farming System and Application of IoT Gateway. In Computational Science/Intelligence and Applied Informatics; Lee, R., Ed.; Springer International Publishing: Cham, Switzerland, 2019; pp. 49-58. [CrossRef]

3. Koksal, O.; Tekinerdogan, B. Architecture design approach for IoT-based farm management information systems. Precis. Agric. 2018, 20, 926-958. [CrossRef]

4. Kamilaris, A.; Gao, F.; Prenafeta-Boldu, F.X.; Ali, M.I. Agri-IoT: A semantic framework for Internet of Things-enabled smart farming applications. In Proceedings of the 2016 IEEE 3rd World Forum on Internet of Things (WF-IoT), Reston, VA, USA, 12-14 December 2016; pp. 442-447. [CrossRef]

5. Ferrandez, J.; Manuel García-Chamizo, J.; Nieto-Hidalgo, M.; Mora-Pascual, J.; Mora-Martínez, J. Developing Ubiquitous Sensor Network Platform Using Internet of Things: Application in Precision Agriculture. Sensors 2016, 16, 1141. [CrossRef] [PubMed]

6. Vasisht, D.; Kapetanovic, Z.; Won, J.; Jin, X.; Chandra, R.; Sinha, S.; Kapoor, A.; Sudarshan, M.; Stratman, S. FarmBeats: An IoT Platform for Data-Driven Agriculture. In Proceedings of the 14th USENIX Symposium on Networked Systems Design and Implementation (NSDI 17), Boston, MA, USA, 27-29 March 2017; USENIX Association: Berkeley, CA, USA, 2017; pp. 515-529;

7. Ahmed, N.; De, D.; Hussain, I. Internet of Things (IoT) for Smart Precision Agriculture and Farming in Rural Areas. IEEE Internet Things J. 2018, 5, 4890-4899. [CrossRef]

8. Ji, M.; Yoon, J.; Choo, J.; Jang, M.; Smith, A. LoRa-based Visual Monitoring Scheme for Agriculture IoT. In Proceedings of the 2019 IEEE Sensors Applications Symposium (SAS), Sophia Antipolis, France, 11-13 March 2019; pp. 1-6. [CrossRef]

9. Ferrandez, J.; Manuel García-Chamizo, J.; Nieto-Hidalgo, M.; Mora-Martínez, J. Precision Agriculture Design Method Using a Distributed Computing Architecture on Internet of Things Context. Sensors 2018, 18, 1731. [CrossRef] [PubMed]

10. Triantafyllou, A.; Sarigiannidis, P.; Lagkas, T.D. Network Protocols, Schemes, and Mechanisms for Internet of Things (IoT): Features, Open Challenges, and Trends. Wirel. Commun. Mob. Comput. 2018, 2018, 5349894. [CrossRef]

11. Triantafyllou, A.; Tsouros, D.C. ; Sarigiannidis, P.; Bibi, S. An Architecture model for Smart Farming. In Proceedings of the 15th International Conference on Distributed Computing in Sensor Systems (DCOSS), Santorini Island, Greece, 29-31 May 2019; pp. 385-392. [CrossRef]

12. International Organization for Standardization; ISO/IEC 7498-1: The Basic Model; Technical Report; 1994; ISO Central Secretariat, Chemin de Blandonnet 8, CP 401 - 1214 Vernier, Geneva, Switzerland. Available Online: https:/ / www.iso.org/standard/20269.html (accessed on 5 November 2019).

13. Vuolo, F.; D’Urso, G.; Michele, C.D.; Bianchi, B.; Cutting, M. Satellite-based irrigation advisory services: A common tool for different experiences from Europe to Australia. Agric. Water Manag. 2015, 147, 82-95. [CrossRef]

14. Bauer, J.; Aschenbruck, N. Design and implementation of an agricultural monitoring system for smart farming. In Proceedings of the 2018 IoT Vertical and Topical Summit on Agriculture-Tuscany (IOT Tuscany), Tuscany, Italy, 8-9 May 2018; pp. 1-6. [CrossRef] 
15. Calera, A.; Campos, I.; Osann, A.; D’Urso, G.; Menenti, M. Remote Sensing for Crop Water Management: From ET Modelling to Services for the End Users. Sensors 2017, 17, 1104. [CrossRef] [PubMed]

16. Petropoulos, G.P.; Srivastava, P.K.; Piles, M.; Pearson, S. Earth Observation-Based Operational Estimation of Soil Moisture and Evapotranspiration for Agricultural Crops in Support of Sustainable Water Management. Sustainability 2018, 10, 181. [CrossRef]

17. Anzola, J.; García Díaz, V.; Jiménez, A. WSN analysis in grid topology for potato crops for IoT. In Proceedings of the 4th Multidisciplinary International Social Networks Conference, Bangkok, Thailand, 17-19 July 2017; pp. 1-7. [CrossRef]

18. Sarigiannidis, P.; Lagkas, T.; Bibi, S.; Ampatzoglou, A.; Bellavista, P. Hybrid 5G optical-wireless SDN-based networks, challenges and open issues. IET Netw. 2017, 6, 141-148. [CrossRef]

19. Jawad, H.M.; Nordin, R.; Gharghan, S.K.; Jawad, A.M.; Ismail, M.; Abu-AlShaeer, M.J. Power Reduction with Sleep/Wake on Redundant Data (SWORD) in a Wireless Sensor Network for Energy-Efficient Precision Agriculture. Sensors 2018, 18, 3450. [CrossRef] [PubMed]

20. Kone, C.T.; Hafid, A.; Boushaba, M. Performance Management of IEEE 802.15.4 Wireless Sensor Network for Precision Agriculture. IEEE Sens. J. 2015, 15, 5734-5747. [CrossRef]

21. Du, K.; Sun, Z.; Zheng, F.; Chu, J.; Ma, J. Monitoring System for Wheat Meterological Disasters using Wireless Sensor Networks. In Proceedings of the 2017 ASABE Annual International Meeting, Spokane, WA, USA, 16-19 July 2017. [CrossRef]

22. Heble, S.; Kumar, A.; Prasad, K.V.V.D.; Samirana, S.; Rajalakshmi, P.; Desai, U.B. A low power IoT network for smart agriculture. In Proceedings of the 2018 IEEE 4th World Forum on Internet of Things (WF-IoT), Singapore, 5-8 February 2018; pp. 609-614. [CrossRef]

23. Anurag, D.; Roy, S.; Bandyopadhyay, S. Agro-sense: Precision agriculture using sensor-based wireless mesh networks. In Proceedings of the 2008 First ITU-T Kaleidoscope Academic Conference-Innovations in NGN: Future Network and Services, Geneva, Switzerland, 12-13 May 2008; pp. 383-388. [CrossRef]

24. El-kader, S.M.A.; El-Basioni, B.M.M. Precision farming solution in Egypt using the wireless sensor network technology. Egypt. Inform. J. 2013, 14, 221-233. [CrossRef]

25. Aquino-Santos, R.; González, A.; Edwards-Block, A.; Virgen Ortíz, R. Developing a New Wireless Sensor Network Platform and Its Application in Precision Agriculture. Sensors 2011, 11, 1192-1211. [CrossRef] [PubMed]

26. Chen, Y.; Chanet, J.P.; Hou, K.M.; Shi, H.; de Sousa, G. A Scalable Context-Aware Objective Function (SCAOF) of Routing Protocol for Agricultural Low-Power and Lossy Networks (RPAL). Sensors 2015, 15, 19507-19540. [CrossRef] [PubMed]

27. Weber, P.; Jäckle, D.; Rahusen, D.; Sikora, A. IPv6 over LoRaWAN ${ }^{\mathrm{TM}}$. In Proceedings of the 2016 3rd International Symposium on Wireless Systems within the Conferences on Intelligent Data Acquisition and Advanced Computing Systems (IDAACS-SWS), Offenburg, Germany, 26-27 September 2016; pp. 75-79. [CrossRef]

28. Sobral, J.V.V.; Rodrigues, J.J.P.C.; Rabêlo, R.A.L.; Saleem, K.; Furtado, V. LOADng-IoT: An Enhanced Routing Protocol for Internet of Things Applications over Low Power Networks. Sensors 2019, 19, 150. [CrossRef] [PubMed]

29. Tukade, T.M.; Banakar, R. Data transfer protocols in IoT-An overview. Int. J. Pure Appl. Math. 2018, 118, 121-138.

30. Mihini/M3DA Specification. Available Online: https://wiki.eclipse.org/Mihini/M3DA_Specification (accessed on 5 November 2019).

31. OMA LightweightM2M. Available Online: https://www.omaspecworks.org/what-is-oma-specworks/iot/ lightweight-m2m-lwm2m/ (accessed on 5 November 2019).

32. oneM2M-Standards for M2M and the Internet of Things. Available Online: http:/ /www.onem2m.org/ (accessed on 5 November 2019).

33. DDS Foundation. Available Online: https://www.dds-foundation.org/what-is-dds-3/ (accessed on 5 November 2019).

34. FIWARE NGSI. Available Online: https://forge.fiware.org/plugins/mediawiki/wiki/fiware/index.php/ FI-WARE_NGSI_Context_Management_tutorial (accessed on 5 November 2019).

35. OGC Implementation Standards. Available Online: https://www.opengeospatial.org/docs/is (accessed on 5 November 2019). 
36. Eleftherakis, G.; Pappas, D.; Lagkas, T.; Rousis, K.; Paunovski, O. Architecting the IoT Paradigm: A Middleware for Autonomous Distributed Sensor Networks. Int. J. Distrib. Sens. Netw. 2015, 2015, 139735. [CrossRef]

37. Capella, J.V.; Campelo, J.C.; Bonastre, A.; Ors, R. A Reference Model for Monitoring IoT WSN-Based Applications. Sensors 2016, 16, 1816. [CrossRef] [PubMed]

38. Ryu, M.; Yun, J.; Miao, T.; Ahn, I.; Choi, S.; Kim, J. Design and implementation of a connected farm for smart farming system. In Proceedings of the 2015 IEEE SENSORS, Busan, Korea, 1-4 November 2015; pp. 1-4. [CrossRef]

39. Wolfert, S.; Ge, L.; Verdouw, C.; Bogaardt, M.J. Big Data in Smart Farming-A review. Agric. Syst. 2017, 153, 69-80. [CrossRef]

40. Westoby, M.; Brasington, J.; Glasser, N.; Hambrey, M.; Reynolds, J. 'Structure-from-Motion' photogrammetry: A low-cost, effective tool for geoscience applications. Geomorphology 2012, 179, 300-314. [CrossRef]

41. Huang, Y.; Reddy, K.N.; Fletcher, R.S.; Pennington, D. In Proceedings of the UAV Low-Altitude Remote Sensing for Precision Weed Management. Weed Technol. 2018, 32, 2-6. [CrossRef]

42. Huang, Y.; Chen, Z.X.; Tao, Y.U.; Huang, X.Z.; Gu, X.F. Agricultural remote sensing big data: Management and applications. J. Integr. Agric. 2018, 17, 1915-1931. [CrossRef]

43. Keswani, B.; Mohapatra, A.G.; Mohanty, A.; Khanna, A.; Rodrigues, J.J.P.C.; Gupta, D.; de Albuquerque, V.H.C. Adapting weather conditions based IoT enabled smart irrigation technique in precision agriculture mechanisms. Neural Comput. Appl. 2019, 31, 277-292. [CrossRef]

44. Hamouda, Y.E.M.; Msallam, M.M. Smart heterogeneous precision agriculture using wireless sensor network based on extended Kalman filter. Neural Comput. Appl. 2019, 31, 5653-5669. [CrossRef]

45. Cambra, C.; Sendra, S.; Lloret, J.; Garcia, L. An IoT service-oriented system for agriculture monitoring. In Proceedings of the 2017 IEEE International Conference on Communications (ICC), Paris, France, 21-25 May 2017; pp. 1-6. [CrossRef]

46. Yoo, S.; Kim, J.; Kim, T.; Ahn, S.; Sung, J.; Kim, D. A2S: Automated Agriculture System based on WSN. In Proceedings of the 2007 IEEE International Symposium on Consumer Electronics, Irving, TX, USA, 20-23 June 2007; pp. 1-5. [CrossRef]

47. Lagkas, T.; Argyriou, V.; Bibi, S.; Sarigiannidis, P. UAV IoT Framework Views and Challenges: Towards Protecting Drones as "Things". Sensors 2018, 18, 4015. [CrossRef] [PubMed]

48. Sharma, G.; Bala, S.; Verma, A.K. Security Frameworks for Wireless Sensor Networks—Review. Procedia Technol. 2012, 6, 978-987. [CrossRef]

49. Pu, C.Energy Depletion Attack Against Routing Protocol in the Internet of Things. In Proceedings of the 2019 16th IEEE Annual Consumer Communications Networking Conference (CCNC), Las Vegas, NV, USA, 11-14 January 2019; pp. 1-4. [CrossRef]

50. Savarimuthu, N.; Vijayalakshmi, K.; Padmapriya, V. A Review of Network Layer Attacks and Countermeasures in WSN. IJESC 2018, 10. [CrossRef]

51. Beltran, V.; Skarmeta, A.F. Overview of Device Access Control in the IoT and its Challenges. IEEE Commun. Mag. 2019, 57, 154-160. [CrossRef]

52. Kumar, R.; Singh, V.; Devi, K.; Sharma, M.; Singh, M.; Ahuja, P. State of Art of Saffron (Crocus sativus L.) Agronomy: A Comprehensive Review. Food Rev. Int. 2008, 25, 44-85. [CrossRef]

53. Melfou, K.; Loizou, E.; Oxouzi, E.; Papanagiotou, E. Economic Performance of Quality Labeled Saffron in Greece. Procedia Econ. Financ. 2015, 24, 419-425. doi:10.1016/S2212-5671(15)00698-X. [CrossRef]

54. van Evert, F.; Gaitán-Cremaschi, D.; Fountas, S.; Kempenaar, C. Can precision agriculture increase the profitability and sustainability of the production of potatoes and olives? Sustainability 2017, 9, 1863. [CrossRef]

55. Erickson, B.; Widmar, D.A. 2015 Precision Agricultural Services Dealership Survey Results; Purdue University: West Lafayette, IN, USA, 2015

56. Baio, F.; Silva, S.; Camolese, H.; Neves, D. Financial analysis of the investment in precision agriculture techniques on cotton crop. Eng. Agrícola 2017, 37, 838-847. [CrossRef]

(C) 2019 by the authors. Licensee MDPI, Basel, Switzerland. This article is an open access article distributed under the terms and conditions of the Creative Commons Attribution (CC BY) license (http:/ / creativecommons.org/licenses/by/4.0/). 